A 203

\title{
Blood Cell Motions and Interactions in Microchannels
}

\author{
Rui LIMA ${ }^{1,2}$, Takuji ISHIKAWA ${ }^{1}$, Hiroki FUJIWARA ${ }^{1}$, Motohiro TAKEDA ${ }^{1,3}$, Yohsuke IMAI ${ }^{1}$, \\ Ken-ichi TSUBOTA ${ }^{1}$, Shigeo WADA ${ }^{4}$, Takami YAMAGUCHI ${ }^{1}$ \\ 1) Dept. Bioeng. \& Robotics, Grad. Sch. Eng., Tohoku Univ., 6-6-01 Aoba, 980-8579 Sendai, Japan. \\ 2) Dept. Mechanical Tech., ESTiG, Braganca Polyt., C. Sta. Apolonia, 5301-857 Braganca, Portugal. \\ 3) Div. Surgical Oncology, Grad. Sch. Medicine, Tohoku Univ., 2-1 Seiryo-machi, Aoba-ku, 980-8575 Sendai, Japan. \\ 4) Dept. Mechanical Science and Bioeng., Grad. Sch. Eng., Osaka Univ., Toyonaka, 560-8531 Osaka, Japan.
}

\begin{abstract}
Detailed knowledge on the motions and interactions of individual blood cells flowing in microchannels is essential to provide a better understanding on the blood rheological properties and disorders in microvessels. This paper presents the ability of a confocal micro-PTV system to track red blood cells (RBCs) through a $100 \mu \mathrm{m}$ circular glass microchannel. The technique consists of a spinning disk confocal microscope, high speed camera and a diode-pumped solid state (DPSS) laser combined with a single particle tracking (SPT) software (MtrackJ). Detailed measurements on the motions of RBCs were measured at different haematocrits (Hct). Our results show clearly that this technique can provide detailed information about microscale disturbance effects caused by the blood cells.
\end{abstract}

Keywords: Microcirculation, Red blood cell, Confocal micro-PIV/PTVD.

\section{INTRODUCTION}

Blood flow in microvessels depends strongly on the motion, deformation and interaction of red blood cells (RBCs). Several studies on both individual and concentrated RBCs have already been performed in the past $[1,2]$. However, all studies used conventional microscopes and also ghost cells to obtain visible trace RBCs at high concentration suspension of blood cells. Recently, considerable progress in the development of confocal microscopy and consequent advantages of this microscope over the conventional microscopes have led to an emerging technique known as confocal micro-PIV [3, 4]. This technique combines the conventional PIV system with a spinning disk confocal microscope (SDCM).

The main purpose of this paper is to evaluate the ability of a confocal micro-PTV system to measure individual RBCs at different haematocrits (Hct) through a $100 \mu \mathrm{m}$ circular glass microchannel.

\section{MATERIALS AND METHODS}

\subsection{Working fluids and microchannel}

The main working fluids used in this study were : dextran 40 (Dx40) containing about $3 \%(3 \mathrm{Hct})$ and $20 \%$ (14Hct) of human red blood cells (RBCs). The blood was collected from a healthy adult volunteer, where ethylenediaminetetraacetic acid
(EDTA) was added to prevent coagulation. The RBCs where separated from the bulk blood by centrifugation (1500 RPM for 5 minutes) and aspiration of the plasma and buffy coat and then washed twice with physiological saline (PS). The washed $\mathrm{RBCs}$ were labeled with a fluorescent cell tracker (CM-Dil, Molecular Probes) and then diluted with Dx40 to make up the required $\mathrm{RBCs}$ concentration by volume. All blood samples were stored hermetical at $4^{\circ} \mathrm{C}$ until the experiment was performed at controlled temperature of about $37^{\circ} \mathrm{C}$.

The microchannel used in this study was a circular borosilicate glass $(100 \mu \mathrm{m}$ in diameter $)$ fabricated by Vitrocom .

\subsection{Confocal micro-PTV experimental set-up}

The confocal micro-PTV system consists of an inverted microscope (IX71, Olympus) combined with a confocal scanning unit (CSU22, Yokogawa), a diode-pumped solid state (DPSS) laser (Laser Quantum Ltd) with an excitation wavelength of $532 \mathrm{~nm}$ and a high-speed camera (Phantom v7.1) (see Figure 1). The microchannel was placed on the stage of the inverted microscope where the flow rate of the working fluids was kept constant by using a syringe pump (KD Scientific Inc.). A thermo plate controller (Tokai Hit) was set to $37^{\circ} \mathrm{C}$. The confocal images were captured with a 
resolution of $640 \times 480$ pixels, at a rate of 100 frames $/ \mathrm{s}$ and then the recorded images were evaluated in Image J (NIH) [6] by using a manual tracking MTrackJ [7] plugin.

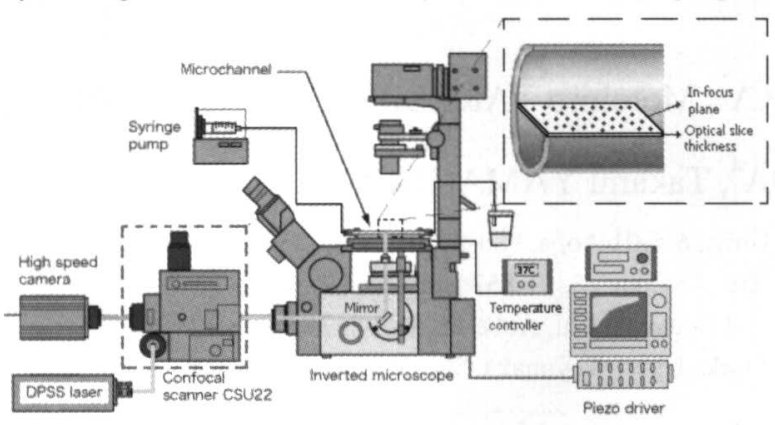

Fig.1 Experimental set-up.

\subsection{RBC radial displacement}

The radial displacements $(\Delta \mathrm{R})$ of the tracked RBCs were determined by using a cumulative radial displacement, given by:

$\Delta R=\sum_{i=0}^{n}\left|R_{0}-R_{i}\right|$

where $R_{0}$ is the initial radial position and $R_{i}$ is the cumulative radial displacement for a defined time interval.

\section{RESULSTS AND DISCUSSION}

Figure 2 shows the streamlines of two-RBC interactions around the plasma layer at $\gamma \sim 16 \mathrm{~s}^{-1}$. This figure shows clearly the radial disturbance effect due to the collision with a neighboring $\mathrm{RBC}$.

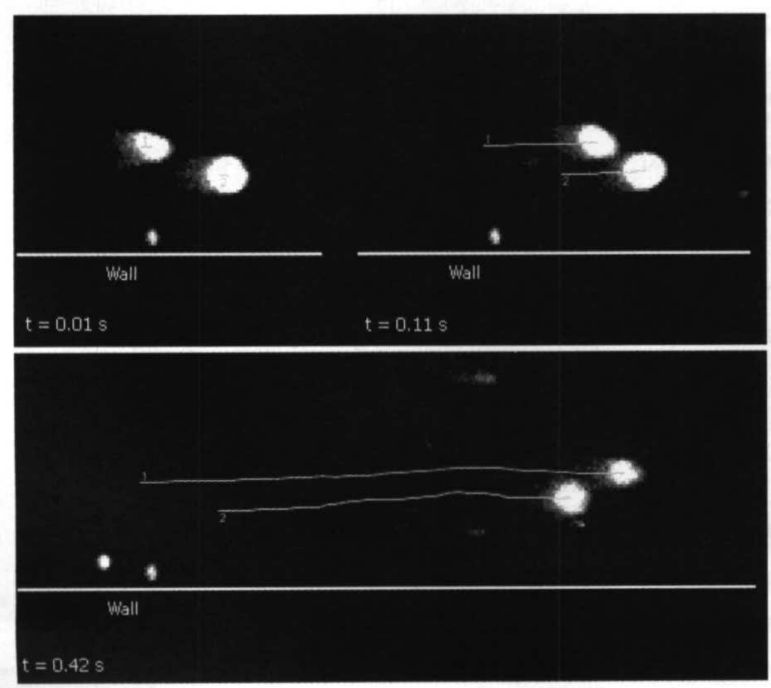

Fig. 2 RBC interactions (40× objective lens).

Figure 3 shows the radial displacement of a RBC (RBCint) that have interacted with a neighboring RBC. Additionally, it is also shown the $\triangle \mathrm{R}$ of a $\mathrm{RBC}$ (RBCnoInt) with any appreciable interaction at $3 \%$ Hct. These results show clearly the fluid-dynamical interactions effect on the motion of RBCs flowing in concentrated suspension of blood cells. For the selected $\mathrm{RBC}$ at $20 \% \mathrm{Hct}$, the $\triangle \mathrm{R}$ has increased of about 2 $\mu \mathrm{m}$ due to the hydrodynamic interactions.

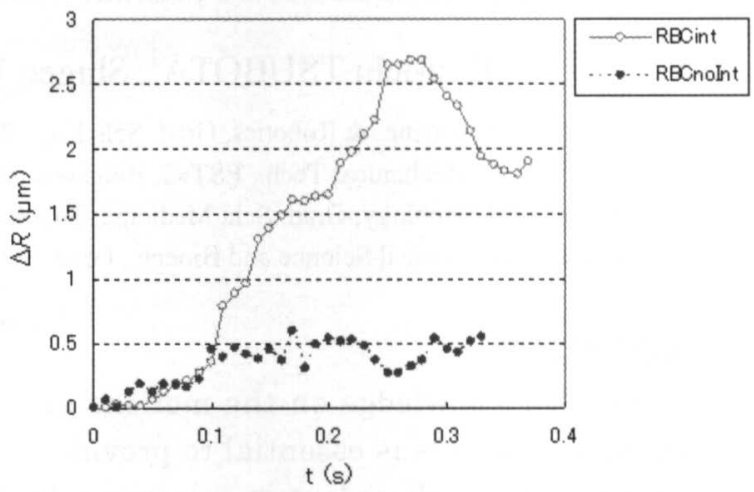

Fig.3 Comparison of the radial displacement $(\triangle \mathrm{R})$ between a $\mathrm{RBC}$ with interactions $(20 \% \mathrm{Hct})$ and $\mathrm{RBC}$ with no interactions ( $3 \% \mathrm{Hct}$ ).

The present study demonstrates that at normal Htcs, the RBC streamlines are continuously diverted and consequently the hydrodynamic interactions introduce disturbances to the blood flow at a microscopic level. The proposed confocal micro-PTV system has the ability to obtain both qualitative and quantitative measurements in flowing blood at concentrate suspensions. We believed that this system will provide a powerful tool to obtain further insight onto the complex flow behavior of blood in microcirculation.

\section{REFERENCES}

1) Goldsmith, H.: Red cell motions and wall interactions in tube flow, Federation Proceedings, Vol. 30, No.5 (1971) pp. 1578-1588.

2) Goldsmith, H. and Marlow J.: Flow behavior of erythrocytes. II. Particles motions in concentrated suspensions of ghost cells, Journal of Colloid and Interface Science, Vol. 71, No. 2 (1979) pp. 383-407.

3) Lima, R., et al.: Confocal micro-PIV measurements of three dimensional profiles of cell suspension flow in a square microchannel, Meas. Sci. Tech., Vol. 17, (2006) pp. 797. 808.

4) Lima, R., et al., In vitro confocal micro-PIV measurements of blood flow in a square microchannel: the effect of the haematocrit on instantaneous velocity profiles, J. Biomech., (in press).

5) Abramoff, M., et al.: Image processing with lmageJ, Biophotonics International, Vol. 11, No. 7 (2004) pp. 36-42.

6) Meijering, E., et al.: Tracking in Molecular Bioimaging,, IEEE Signal Processing Magazine, Vol. 23, No. 3 (2006) pp. 46-53. 